\title{
Three-dimensional bipedal walking control using Divergent Component of Motion
}

\author{
Johannes Englsberger, Christian Ott, Alin Albu-Schäffer
}

\begin{abstract}
In this paper, we extend the Divergent Component of Motion (DCM, also called 'Capture Point') to 3D. We introduce the "Enhanced Centroidal Moment Pivot point" (eCMP) and the "Virtual Repellent Point" (VRP), which allow for the encoding of both direction and magnitude of the external (e.g. leg) forces and the total force (i.e. external forces plus gravity) acting on the robot. Based on eCMP, VRP and DCM, we present a method for real-time planning and control of DCM trajectories in 3D. We address the problem of underactuation and propose methods to guarantee feasibility of the finally commanded forces. The capabilities of the proposed control framework are verified in simulations.
\end{abstract}

\section{INTRODUCTION}

The basic challenge in robotic locomotion research is how to maneuver the robot (or more precisely: its Center of Mass $(\mathrm{CoM})$ ) from one point $\mathrm{A}$ in space to another point $\mathrm{B}$. The CoM dynamics is decoupled from the rest of the robot dynamics [1] and is only affected by gravity and the external (e.g. leg) forces (conservation of momentum law):

$$
\ddot{\boldsymbol{x}}=\frac{1}{m} \boldsymbol{F}=\frac{1}{m}\left(\boldsymbol{F}_{g}+\boldsymbol{F}_{\text {ext }}\right),
$$

where $\ddot{\boldsymbol{x}}=[\ddot{x} \ddot{y} \ddot{z}]^{T}$ is the CoM acceleration, $\boldsymbol{F}_{\text {ext }}$ is the sum of all external forces, $\boldsymbol{F}_{g}=m\left[\begin{array}{ll}0 & 0-g\end{array}\right]^{T}$ is the gravitational force and $g$ is the gravitational constant. As the CoM dynamics is a second order dynamics, the CoM position at a given point $t$ in time can be derived by double integration of the CoM acceleration. One difficulty arises from the fact, that there exists an infinite number of different timetransient total forces $\boldsymbol{F}$ for which the CoM motion would fulfill the constraints $\boldsymbol{x}_{0}=\boldsymbol{x}_{A}$ and $\boldsymbol{x}_{\text {end }}=\boldsymbol{x}_{B}$. This poses the problem that no unique solution to the problem exists. Additionally, in order to be physically feasible, the line of action of the sum of external forces acting on the robot has to pass through the base of support (e.g. convex hull of robot's stance feet). The use of a standard tracking controller of the form $\boldsymbol{F}=k_{p}\left(\boldsymbol{x}_{d}-\boldsymbol{x}\right)+k_{d}\left(\dot{\boldsymbol{x}}_{d}-\dot{\boldsymbol{x}}\right)+m \ddot{\boldsymbol{x}}_{d}$ disregards this physical constraint and is therefore not well suited for bipedal walking control. One idea to solve this feasibility problem is the following: Instead of checking if the lines of action of the leg forces intersect the base of support, a point of intersection (focus of all lines of action of external forces) is designed and related to a correlating force via an appropriate force law. The Linear Inverted Pendulum (LIP) model [2] directly follows this idea, its torque-free base joint

Institute of Robotics and Mechatronics, German Aerospace Center, 82234 Wessling, Germany. E-mail: johannes.englsberger@dlr.de

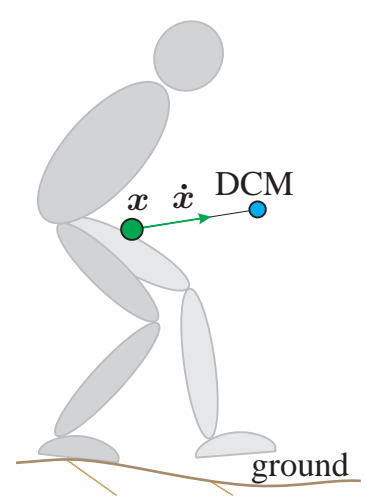

Fig. 1. Divergent Component of Motion (DCM) as point in 3D space

being the mentioned focus point. Using (1) and the equation for the horizontal LIP acceleration, we can write the total three-dimensional force acting on the CoM as

$$
\boldsymbol{F}_{L I P}=\underbrace{\left[\begin{array}{c}
0 \\
0 \\
-m g
\end{array}\right]}_{\boldsymbol{F}_{g}}+\underbrace{m \frac{g}{z-p_{z}}(\boldsymbol{x}-\boldsymbol{p})}_{\boldsymbol{F}_{l e g}}=m \omega^{2}\left[\begin{array}{c}
x-p_{x} \\
y-p_{y} \\
0
\end{array}\right],
$$

where $\boldsymbol{F}_{g}$ and $\boldsymbol{F}_{\text {leg }}$ are the gravitational and leg force, $\boldsymbol{x}=\left[\begin{array}{lll}x & y & z\end{array}\right]^{T}$ denotes the position of the CoM, $\boldsymbol{p}=\left[\begin{array}{ll}p_{x} & p_{y} \\ p_{z}\end{array}\right]^{T}$ are the LIP base joint coordinates and $1 / \omega$ is the timeconstant of the LIP dynamics. The vertical components of $\boldsymbol{F}_{g}$ and $\boldsymbol{F}_{\text {leg }}$ cancel each other, so that $\ddot{z}=0$ holds. Note that the term $\frac{m g}{z-p_{z}}=m \omega^{2}$ is constant and might even be combined into one single constant. Due to the torque-free base and the point mass assumption, all forces pass through the CoM and the LIP base joint, which therefore can be seen as a focus point of all (lines of action of) leg forces. When the LIP is used for bipedal walking control, the Zero Moment Point (ZMP, [3]) is assumed to be equivalent to the LIP base joint $\boldsymbol{p}$ and is designed to be within the foot (e.g. in the foot center). In a real robot, the resulting ZMPs generally deviate from the planned focus points due to the model inaccuracies, but often not far enough to make the robot tilt and fall. This way, preplanning a feasible set of focus points (ZMP's) is a successful method for bipedal gait generation and control: Stephens and Atkeson [4] present a Model Predictive Control (MPC) method for step adjustment and push recovery. Wieber [5] proposes a trajectory free linear MPC scheme, allowing for compensation of strong perturbations. Nishiwaki and Kagami [6] generate dynamically stable walking patterns 
by frequently updating a preview controller. Sugihara [7] introduces the Best COM-ZMP Regulator facilitating step adjustment of bipedal robots. Kajita et al. [8] demonstrate outdoor walking on uneven pavement.

Several previous works, such as [9]-[17], propose the idea of splitting CoM dynamics into a stable and an unstable part (traditionally based on LIP model dynamics). The unstable part of the dynamics has been referred to as 'Extrapolated Center of Mass' by Hof et al. [9], '(instantaneous) Capture Point' by Pratt and Koolen et al. [10]-[12] and 'Divergent Component of Motion' (DCM) by Takenaka et al. [13]. Throughout this paper, we use the notation of Takenaka et al. and the symbol $\boldsymbol{\xi}$ to represent the DCM. In our previous works [14], [15], motivated by the works of Pratt et al., we used the term 'Capture Point' for the DCM. In this paper, we depict a significant difference between the Capture Point (defined by Pratt et al. as the point on the ground, to which the robot has to step to come to a stop asymptotically) and the Divergent Component of Motion, as the DCM is not restricted to the ground plane, but can be interpreted as three-dimensional state (see fig. 1). For considerations in 2D (constant CoM height), Capture Point and DCM (projected to floor) are equivalent, but not so for 3D.

The use of the LIP model for bipedal walking control is restricted to horizontal motions of the CoM $(z=$ const $)$. This motivates the derivation of methods, which are not limited to constant CoM and floor height. Zhao and Sentis [18] introduce the Prismatic Inverted Pendulum dynamics and solve it via numerical integration, allowing for threedimensional foot placement planning on uneven ground surfaces. Yet, in their method the lateral foot-placement cannot be predefined, but is dependent on the sagittal dynamics and the desired CoM Surface. Additionally, the method is restricted to ground surfaces with laterally constant heights ("roughness" of terrain only in forward direction).

In this paper, we derive - motivated by the capabilities of Capture Point control [15] - a method for bipedal gait planning and control on uneven terrain, facilitated by the use of the linear properties of the DCM dynamics and suffering from none of the afore mentioned restrictions.

\section{THEORY}

\section{A. Divergent Component of Motion (DCM)}

Motivated by the performance of Capture Point control in [14], [15] and by the definition of the Capture Point (or two-dimensional DCM) in [10], [13], we introduce the threedimensional Divergent Component of Motion (DCM) as

$$
\boldsymbol{\xi}=\boldsymbol{x}+b \dot{\boldsymbol{x}},
$$

where $\boldsymbol{\xi}=\left[\boldsymbol{\xi}_{x}, \boldsymbol{\xi}_{y}, \boldsymbol{\xi}_{z}\right]^{T}$ is the DCM, $\boldsymbol{x}=[x, y, z]^{T}$ and $\dot{\boldsymbol{x}}=$ $[\dot{x}, \dot{y}, \dot{z}]^{T}$ are the CoM position and velocity and $b>0$ is the time-constant of the DCM dynamics. Note that (3) is a general transformation and (just like all subsequent derivations) not restricted to the LIP or other simplified models. Also note the similarity of (3) to the state transformation proposed by
Slotine and Li [19] used for adaptive control. In contrast to the definition of the Capture Point in [9]-[15], in equation (3) the DCM is defined as a point that lies at a certain distance in front of the CoM (in its current moving direction), i.e. it is generally not located on the ground but somewhere in space. By reordering (3), we can derive the CoM dynamics

$$
\dot{\boldsymbol{x}}=-\frac{1}{b}(\boldsymbol{x}-\boldsymbol{\xi}) .
$$

This shows that the CoM has a stable first order dynamics for $b>0$ ( $\rightarrow$ it follows the DCM). By differentiating (3) and inserting (4) and (1), we find the DCM dynamics

$$
\dot{\boldsymbol{\xi}}=\dot{\boldsymbol{x}}+b \ddot{\boldsymbol{x}}=-\frac{1}{b} \boldsymbol{x}+\frac{1}{b} \boldsymbol{\xi}+\frac{b}{m} \boldsymbol{F} .
$$

This shows that $\boldsymbol{F}$ directly influences the DCM dynamics. Note that, due to the linearity of a general (multi-body) robot's CoM dynamics, the equations and control laws, derived in this and all following sections, hold for general freefloating robot models and not only for simplified models, such as telescopic or linear inverted pendulum.

\section{B. Enhanced Centroidal Moment Pivot point (eCMP)}

Generally, a robot is subject to gravity and external forces. As proposed in the introduction, we design external forces being appropriate for the locomotion task while fulfilling the feasibility constraint (CoP in base of support). To simplify this design process, we make use of a force-to-point transformation similar as in the LIP model. Remember that the term $\frac{m g}{z-p_{z}}$ in (2) is constant. This motivates the encoding of external forces in a simple repelling force law (linear dependancy), based on the difference of the CoM and the so called Enhanced Centroidal Moment Pivot point (eCMP):

$$
\boldsymbol{F}_{\text {ext }}=s\left(\boldsymbol{x}-\boldsymbol{r}_{\text {ecmp }}\right),
$$

where $s>0$ is a constant, which we determine later. The eCMP is closely related to the CMP [20], but it is not restricted to be within the foot plane or ground surface. This allows for encoding of not only the direction of the sum of external forces, but also its magnitude. At any time, the CMP can be computed by intersecting the line between the CoM and the eCMP with the ground surface (see fig. 2). The total force acting on the CoM is

$$
\boldsymbol{F}=\boldsymbol{F}_{\text {ext }}+\boldsymbol{F}_{g}=s\left(\boldsymbol{x}-\boldsymbol{r}_{\text {ecmp }}\right)+m \boldsymbol{g}
$$

Inserting (7) into (5), we find the DCM dynamics:

$$
\dot{\boldsymbol{\xi}}=\left(\frac{b s}{m}-\frac{1}{b}\right) \boldsymbol{x}+\frac{1}{b} \boldsymbol{\xi}-\frac{b s}{m} \boldsymbol{r}_{\text {ecmp }}+b \boldsymbol{g} .
$$

This shows that the states $\boldsymbol{x}$ and $\boldsymbol{\xi}$ are coupled in general. Though, by the choice $s=\frac{m}{b^{2}}$, we can decouple the DCM dynamics from the CoM dynamics:

$$
\dot{\boldsymbol{\xi}}=\frac{1}{b} \boldsymbol{\xi}-\frac{1}{b} \boldsymbol{r}_{\text {ecmp }}+b \boldsymbol{g} .
$$

The corresponding equation for the sum of external forces is

$$
\boldsymbol{F}_{\text {ext }}=\frac{m}{b^{2}}\left(\boldsymbol{x}-\boldsymbol{r}_{\text {ecmp }}\right) \text {. }
$$




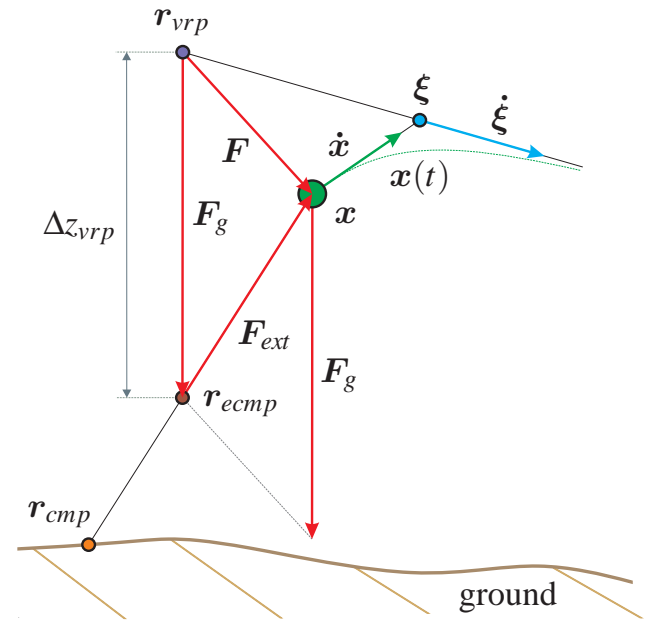

Fig. 2. Force encoding via eCMP and VRP. The figure shows an example 2D case. The VRP encodes the sum of all forces (gravity and external forces) $\boldsymbol{F}$. The Enhanced Centroidal Moment Pivot (eCMP) encodes the external forces $\boldsymbol{F}_{\text {ext }}$ via (16) and is located at a vertical offset $\Delta_{\text {z }} r p$ below the VRP. Note that by means of appropriate scaling (length of force arrow equals $\Delta z_{v r p} /(m g)$ times force magnitude) the forces can geometrically be related to correspondent point distances (comp. to equations (14) and (16)).

\section{Virtual Repellent Point (VRP)}

To simplify (9) even more, we introduce the Virtual Repellent Point (VRP), which is defined as

$$
\boldsymbol{r}_{v r p}=\boldsymbol{r}_{\text {ecmp }}+\left[\begin{array}{lll}
0 & 0 & b^{2} g
\end{array}\right]^{T}=\boldsymbol{r}_{\text {ecmp }}+\left[\begin{array}{lll}
0 & 0 & \Delta z_{v r p}
\end{array}\right]^{T} .
$$

The $x$ - and $y$ - components of the eCMP and the VRP are equal. Their vertical components $z_{e c m p}$ and $z_{v r p}$ differ by $\Delta z_{v r p}=b^{2} g$. Using the definition of the VRP, we can rewrite the DCM dynamics as

$$
\dot{\xi}=\frac{1}{b}\left(\boldsymbol{\xi}-\boldsymbol{r}_{v r p}\right)
$$

This shows that the DCM has an unstable first order dynamics (it is "pushed" by the VRP on a straight line), whereas the CoM follows the DCM with the stable first order dynamics (4) (see fig. 2). The overall open-loop dynamics is

$$
\left[\begin{array}{c}
\dot{\boldsymbol{x}} \\
\dot{\boldsymbol{\xi}}
\end{array}\right]=\left[\begin{array}{cc}
-1 / b & 1 / b \\
0 & 1 / b
\end{array}\right]\left[\begin{array}{l}
\boldsymbol{x} \\
\boldsymbol{\xi}
\end{array}\right]+\left[\begin{array}{c}
0 \\
-1 / b
\end{array}\right] \boldsymbol{r}_{v r p}
$$

Basically, the VRP encodes the effects of gravity and the external forces in one single point. With (7), (10), (11) and $\Delta z_{v r p}=b^{2} g$, we find the correlation between the total force $\boldsymbol{F}$ acting on the CoM (see fig. 2) and the VRP as

$$
\boldsymbol{F}=\frac{m}{b^{2}}\left(\boldsymbol{x}-\boldsymbol{r}_{v r p}\right)=\frac{m g}{\Delta z_{v r p}}\left(\boldsymbol{x}-\boldsymbol{r}_{v r p}\right) .
$$

Figure 3 clarifies the correlations between the eCMP, the CMP and the CoP for general (bipedal) robot dynamics.

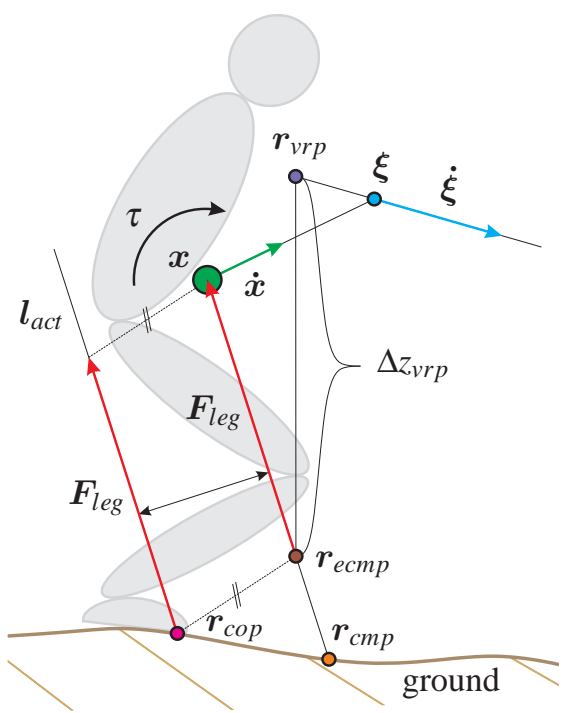

Fig. 3. Point correlations for general robot dynamics. The CMP is found as the intersection of the line CoM-to-eCMP with the ground. The line of action $\boldsymbol{l}_{\text {act }}$ of the leg force can be shifted by means of a torque $\tau$ around the CoM, so that the CoP does generally not coincide with the CMP.

\section{GENERATION OF DCM REFERENCE}

Section II provided all tools, that are necessary to derive a method to (online) plan and track a desired DCM trajectory in 3D. For planning, we make the following assumptions:

- robot's feet are point feet (corresponding to foot centers if robot has finite-sized feet)

- changes in angular momentum $\boldsymbol{L}$ are zero $(\dot{\boldsymbol{L}}=0)$

- instantaneous transitions between left and right single support phases (no double support)

- no impacts during support transitions

The basic idea is to start with a set of $N$ foot positions $\boldsymbol{r}_{f, i}$ (where $i \in\{1, \ldots, N\}$ ) which are placed on a threedimensional ground-surface (see fig 5). For planning, we choose to coincide eCMP and CoP (see fig. 4), so that the corresponding forces always pass through both CoM and planned foot position $\boldsymbol{r}_{f, i}$. This leads to a constant $\mathrm{CoP}$ in case of no perturbations. Using the assumptions from above, a desired DCM trajectory can be derived as follows: Firstly, given a desired eCMP-to-VRP height difference $\Delta z_{\text {vrp }}$, we find the correspondent desired VRPs (see fig 5) with (11) as

$$
\boldsymbol{r}_{v r p, d, i}=\boldsymbol{r}_{f, i}+\left[\begin{array}{lll}
0 & 0 & \Delta z_{v r p}
\end{array}\right]^{T}
$$

With (11), we find the time-constant of the DCM dynamics as $b=\sqrt{\Delta z_{v r p} / g}$, so that (10) can be expressed as

$$
\boldsymbol{F}_{\text {ext }}=\boldsymbol{F}_{\text {leg }}=\frac{m g}{\Delta z_{v r p}}\left(\boldsymbol{x}-\boldsymbol{r}_{\text {ecmp }}\right) .
$$

Here we replaced the sum of external forces $\boldsymbol{F}_{\text {ext }}$ by the leg force $\boldsymbol{F}_{l e g}$, as in this paper we only consider the leg force for planning and control. For $\Delta z_{v r p}>0$ the term $\frac{m g}{\Delta z_{v r p}}$ is positive so that the unilaterality constraint (only "pushing" leg forces) is fulfilled. Note that in steady state (e.g. robot's CoM 




Fig. 4. For planning, the eCMP is designed to coincide with the point foot (or foot center) which along with the assumption $\dot{\boldsymbol{L}}=0$ leads to a constant focus point, through which all force lines pass.

balanced over one foot) $\boldsymbol{x}=\boldsymbol{\xi}=\boldsymbol{r}_{v r p}=\boldsymbol{r}_{\text {ecmp }}+\left[\begin{array}{lll}0 & 0 & \Delta z_{v r p}\end{array}\right]^{T}$ holds, so that $\boldsymbol{F}_{l e g}=\left[\begin{array}{lll}0 & 0 & m g\end{array}\right]$ simply compensates for gravity. To find the desired DCM trajectory from given VRPs, the method presented in [15] is extended to 3D. For a constant VRP, the solution of (12) in time is

$$
\boldsymbol{\xi}(t)=\boldsymbol{r}_{v r p}+e^{t / b}\left(\boldsymbol{\xi}_{0}-\boldsymbol{r}_{v r p}\right),
$$

where $\boldsymbol{\xi}_{0}$ is the DCM at $t=0$. The "internal" step time $t$ is reset at the beginning of each step, i.e. $t \in\left[0, t_{\text {step }}\right]$. Using (17) and constant desired VRPs, we find the desired DCM locations at the end of each step via recursion:

$$
\boldsymbol{\xi}_{d, e o s, i-1}=\boldsymbol{\xi}_{d, i n i, i}=\boldsymbol{r}_{v r p, d, i}+e^{-\frac{t_{s t e p}}{b}}\left(\boldsymbol{\xi}_{d, e o s, i}-\boldsymbol{r}_{v r p, d, i}\right) .
$$

Note that different times per step $t_{\text {step }}$ might be chosen for subsequent steps without loss of generality. Using equation (18), from the final step (after which the robot usually comes to a stop) until the current step, all $\boldsymbol{\xi}_{d, e o s, i}$ as well as the whole future desired trajectory of the DCM (bold blue lines in fig. 5) can be calculated. Taking all $N$ future foot positions into account, we would start with $\boldsymbol{\xi}_{d, e o s, N-1}=\boldsymbol{r}_{v r p, d, N}$ and use (18) to find all final DCMs $\boldsymbol{\xi}_{d, e o s, i}$ until the first (or current) one. In practice, we limit ourselves to the use of the current ( $\boldsymbol{r}_{v r p, d, 1}$, the index 1 always indicates the current step) and next three desired VRPs $\left(\boldsymbol{r}_{v r p, d, 2}, \boldsymbol{r}_{v r p, d, 3}\right.$ and $\left.\boldsymbol{r}_{v r p, d, 4}\right)$ for the calculation of the DCM tracking reference. This reduces the computational effort while the deviation from the trajectory generation using all future VRPs is marginal. Using (17), we derive the desired DCM trajectory in time

$$
\boldsymbol{\xi}_{d}(t)=\boldsymbol{r}_{v r p, d, 1}+e^{\frac{t-t_{s t e p}}{b}}\left(\boldsymbol{\xi}_{d, e o s, 1}-\boldsymbol{r}_{v r p, d, 1}\right)
$$

for $t<t_{\text {step. }}$. This corresponds to the first blue line section $\left(\boldsymbol{\xi}_{d, i n i, 1}\right.$ to $\boldsymbol{\xi}_{d, e o s, 1}$, $\rightarrow$ first step) in fig. 5. Note that $\boldsymbol{\xi}_{\boldsymbol{d}}(0)=\boldsymbol{\xi}_{d, i n i, 1}$ and $\boldsymbol{\xi}_{\boldsymbol{d}}\left(t_{\text {step }}\right)=\boldsymbol{\xi}_{d, e o s, 1}$.



Fig. 5. Planning of DCM trajectory over rough terrain. Note that both the DCM reference trajectory (bold blue lines) and the resulting CoM trajectory (green sinusoidal curve) are three-dimensional, i.e. not constraint to be within a horizontal plane (see also fig. 9). The CoM "automatically" follows the DCM from its initial position $\boldsymbol{x}_{0}$ to the final equilibrium point $\boldsymbol{r}_{v r p, d, N}$.

\section{THREE-DIMENSIONAL DCM TRACKING CONTROL}

To track the desired DCM trajectory, we are looking for a controller of the following basic characteristics

$$
\underbrace{\dot{\boldsymbol{\xi}}-\dot{\boldsymbol{\xi}}_{d}}_{\dot{e}_{\xi}}=-k \underbrace{\left(\boldsymbol{\xi}-\boldsymbol{\xi}_{d}\right)}_{\boldsymbol{e}_{\xi}},
$$

which is stable for $k>0$ (DCM error $\boldsymbol{e}_{\xi}$ converges asymptotically). This desired dynamics can be realized exactly by a DCM tracking control law of the form

$$
\boldsymbol{r}_{v r p, c}=\boldsymbol{\xi}+k b\left(\boldsymbol{\xi}-\boldsymbol{\xi}_{\boldsymbol{d}}\right)-b \dot{\boldsymbol{\xi}}_{\boldsymbol{d}},
$$

which can be verified by setting $\boldsymbol{r}_{v r p}=\boldsymbol{r}_{v r p, c}$ in (12). This DCM control law returns the specific VRP $\boldsymbol{r}_{v r p, c}$, which realizes the desired tracking behavior ("c" stands for "control"). Note that the DCM control law (21) only stabilizes the unstable part of the dynamics (which is the DCM dynamics), without influencing the naturally stable CoM dynamics (4). The closed loop dynamics has the following form

$$
\left[\begin{array}{c}
\dot{\boldsymbol{x}} \\
\dot{\boldsymbol{\xi}}
\end{array}\right]=\underbrace{\left[\begin{array}{cc}
-1 / b & 1 / b \\
0 & -k
\end{array}\right]\left[\begin{array}{l}
\boldsymbol{x} \\
\boldsymbol{\xi}
\end{array}\right]}_{\text {feedback }}+\underbrace{\left[\begin{array}{ll}
0 & 0 \\
k & 1
\end{array}\right]\left[\begin{array}{l}
\boldsymbol{\xi}_{d} \\
\dot{\boldsymbol{\xi}}_{d}
\end{array}\right]}_{\text {feedforward }} .
$$

The eigenvalues $-1 / b$ and $-k$ of the system matrix are stable for $k>0$ and $b>0$. It has to be noted, that this stability analysis does not take any physical limitations (such as limited base of support) into account (see Sec. (V)). By the use of the proposed control law, the three-dimensional system dynamics are time-invariant, which is a major advantage compared to [15]. In this paper, we use constant foot-positions (and constant desired eCMPs and VRPs accordingly) for planning 


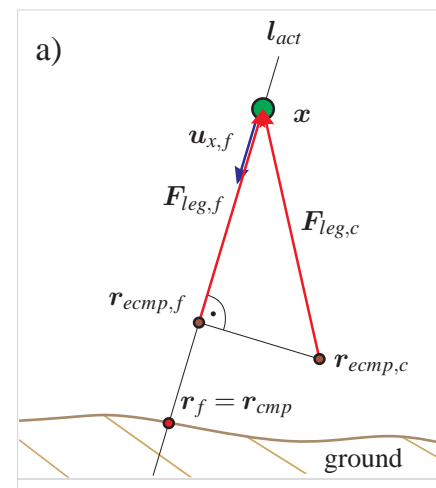

b)

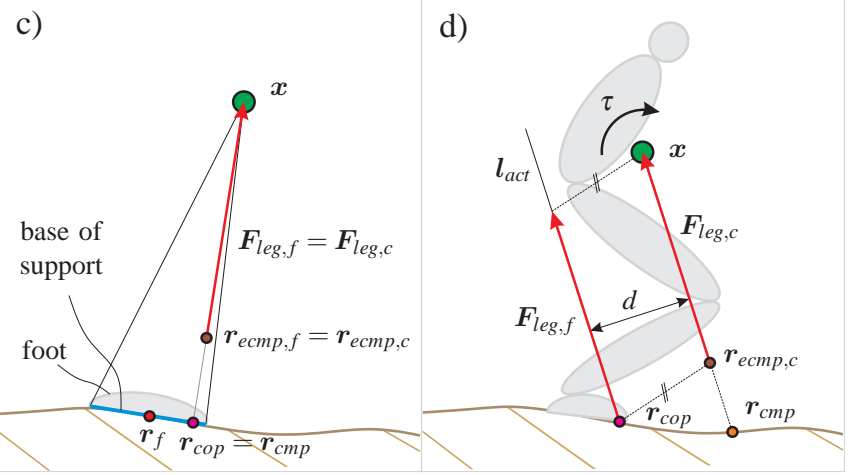

Fig. 6. Mechanisms for producing best feasible leg force. a) projection to feasible direction, b) step adjustment,

c) $\mathrm{CoP}$ modulation in base of support, d) use of angular momentum

(point foot assumption), which with (12) allows us to express the desired DCM velocity in the following form

$$
\dot{\boldsymbol{\xi}}_{d}=\frac{1}{b}\left(\boldsymbol{\xi}_{d}-\boldsymbol{r}_{v r p, d, 1}\right)
$$

Using (23) we simplify the DCM tracking control law (21):

$$
\boldsymbol{r}_{v r p, c}=\boldsymbol{r}_{v r p, d, 1}+(1+k b)(\underbrace{\boldsymbol{\xi}-\boldsymbol{\xi}_{d}}_{\boldsymbol{e}_{\boldsymbol{\xi}}}) .
$$

As the DCM error $\boldsymbol{e}_{\boldsymbol{\xi}}$ converges asymptotically, also the commanded VRP $\boldsymbol{r}_{v r p, c}$ and its corresponding eCMP $\boldsymbol{r}_{e c m p, c}$ converge to their desired values $\left(\boldsymbol{r}_{v r p, d, 1}\right.$ and $\left.\boldsymbol{r}_{e c m p, d, 1}=\boldsymbol{r}_{f, 1}\right)$ asymptotically after a perturbation. Inserting the desired VRP $\boldsymbol{r}_{v r p, c}$ from (24) into (16), we find the desired force as

$$
\boldsymbol{F}_{\text {leg,c }}=\frac{m g}{\Delta z_{v r p}}\left(\boldsymbol{x}-(\underbrace{\boldsymbol{r}_{v r p, c}-\left[\begin{array}{lll}
0 & 0 & \Delta z_{v r p}
\end{array}\right]^{T}}_{\boldsymbol{r}_{\text {ecmp }, c}})\right)
$$

Note that the only equations that are finally needed are (18) and (19) for three-dimensional DCM trajectory generation and (24) and (25) for force-based DCM tracking control. They can easily be computed in real-time on any computer.

Regarding robustness, we found that it increases for shorter times per step $t_{\text {step }}$ and bigger DCM error gains $k$. Foot positions are less relevant, as long as they are reachable by the physical robot.

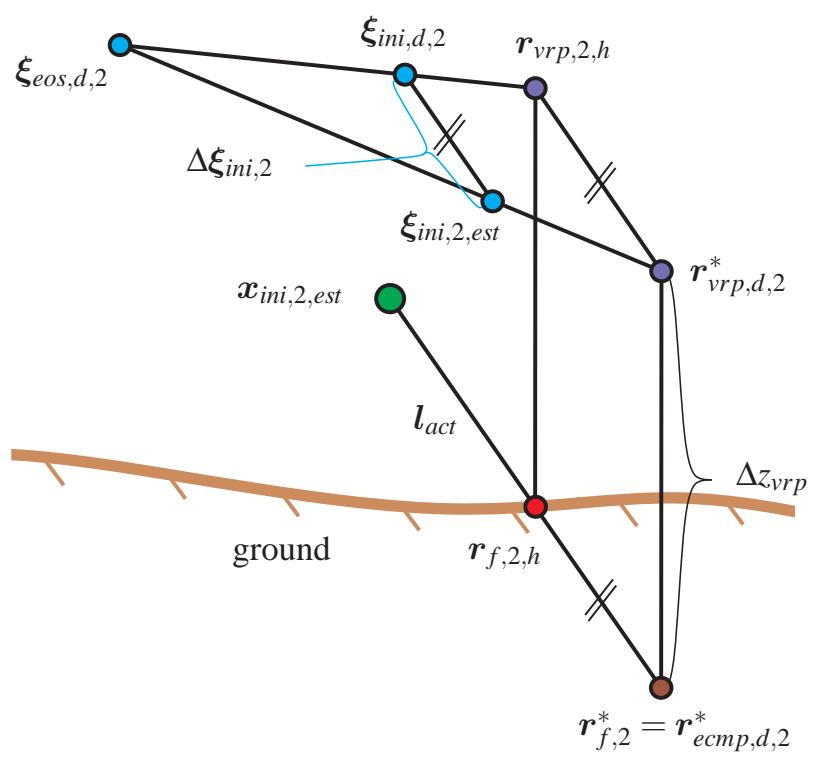

Fig. 7. Heuristics for appropriate step location on 3D ground surface. The index "h" stands for "heuristic". Starting with the estimated DCM $\xi_{n i, 2, e s t}$ at the step transition, the VRP $\boldsymbol{r}_{v r p, d, 2}^{*}$ (and its corresponding eCMP $\boldsymbol{r}_{e c m p, d, 2}^{*}$ $\left(\Delta z_{v r p}\right.$ below it)) is computed, which would shift the DCM from its initial position to the final desired DCM $\xi_{\text {eos,d,2 }}$ within the next stepping time. The intersection of the line CoM-to- $\boldsymbol{r}_{\text {ecmp,d,2 }}^{*}$ with the ground is chosen as heuristic foot location $\boldsymbol{r}_{f, 2, h}$. The desired VRP $\boldsymbol{r}_{v r p, 2, h}$ is found $\Delta z_{v r p}$ further above. With $\boldsymbol{r}_{v r p, 2, h}$ and the final desired DCM $\boldsymbol{\xi}_{e o s, d, 2}$, the desired initial DCM $\boldsymbol{\xi}_{\text {ini, }, 2}$ is computed. The initial commanded eCMP $\boldsymbol{r}_{e c m p, c}$ (not shown; computed from the initial DCM error $\Delta \xi_{\text {ini,2 }}$ via (24) and (11)) is perfectly in the actuated direction (line CoM-to-foot, note parallelisms), which assures good convergence in the beginning of the subsequent step.

\section{Physical Limitations DUE TO UNDERACTUATION AND FEASIBLE CONTROL MECHANISMS}

A general humanoid robot can be modeled as free-floating multi-body system. When walking, it is usually underactuated [1], making bipedal walking control a challenge. In the proposed control method, in the unperturbed case (see Sec. III) the desired leg forces intersect the desired point foot location (or foot center) and the CoM at all times, so that they are feasible. In case of perturbations (e.g. $\dot{\boldsymbol{L}} \neq 0$ ), the forces required to track the desired DCM trajectory (see control laws (24) and (25)) can highly deviate from the originally planned ones and therefore are no longer guaranteed to be feasible. Depending on technical characteristics and current configuration of the robot, there are different methods allowing for feasible force modulation (magnitude and direction):

1) Variation of force magnitude along feasible direction: If the robot is modeled as a point mass with point foot $\left(\rightarrow \boldsymbol{r}_{f}=\boldsymbol{r}_{c m p}=\boldsymbol{r}_{c o p}\right.$ ), the eCMP (and correlating leg force) can be modulated along the direction of the vector $\boldsymbol{u}_{x, f}$ (unit vector pointing from CoM to point foot, see fig. 6 a). This direction is actuated (only for pushing leg forces), whereas the other two spatial directions are unactuated. Thus, the 


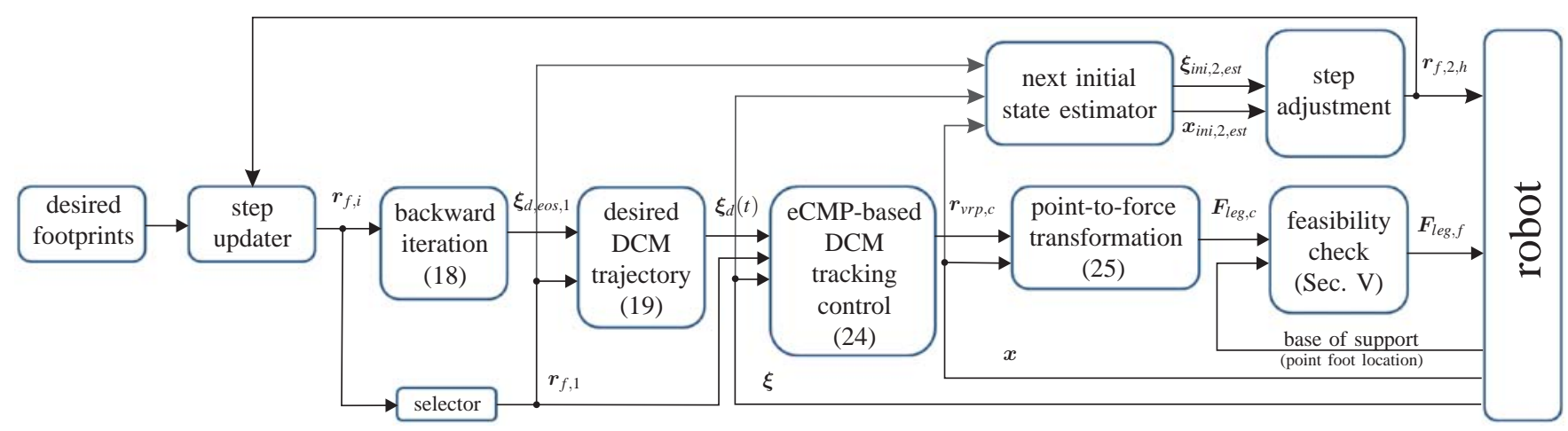

Fig. 8. Overall control algorithm used for Prismatic Inverted Pendulum simulation ( $\rightarrow$ in OpenHRP3 simulation: no step adjustment)

desired eCMP has to be projected to the feasible direction:

$$
\boldsymbol{r}_{e c m p, f}=\boldsymbol{x}+\boldsymbol{u}_{x, f} \boldsymbol{u}_{x, f}^{T}\left(\boldsymbol{r}_{e c m p, c}-\boldsymbol{x}\right)
$$

where $\boldsymbol{r}_{\text {ecmp,f }}$ is the feasible eCMP. To comply with the unilaterality constraint (only pushing leg forces), desired eCMPs "above" the CoM are projected onto the CoM itself, resulting in zero leg force ( $\rightarrow$ free-falling robot).

2) Variation of force direction via step adjustment: Stepping is not only needed for the robot to move from one location to another, but is also a very efficient method for adjusting the leg force direction (see fig. 6 b) and recovering from strong perturbations. The subsequent step position is adjusted so that the expected desired forces can be produced via the resulting $\mathrm{CoP}$ as good as possible. Often, it is difficult to predict, what step location is optimal, so that a heuristic is used. The heuristic used in this paper is described in fig. 7.

3) Variation of force direction via CoP modulation: If the robot has a finite-sized foot (or both legs in contact), it can change the direction of the leg force, as long as the CoP lies within the base of support (see fig. 6 c). If the desired direction lies outside, it has to be projected via (26).

4) Shift of line of action of leg force via torque around CoM: If the robot has rotational inertia available, it can produce a torque around the CoM. That way, the line of action $l_{a c t}$ of the leg force can be shifted into a feasible CoP location (see fig. $6 \mathrm{~d}$ ).

The control strategies for a humanoid robot may combine the different mechanisms described here in order to best possible produce the desired leg force. Note that global stability, as shown for the underlying control laws (24) and (25) (see (22)), can no longer be guaranteed for an underactuated robot.

\section{Simulations}

\section{A. Prismatic Inverted Pendulum simulations (point mass)}

In order to demonstrate the performance of the proposed control framework, we carried out simulations, modeling the robot as Prismatic Inverted Pendulum [18] (point mass with two point feet). Figure 8 shows the overall control framework used for the simulation. We assumed instantaneous, impactfree transitions between left and right single support phases (no double support). Figure 9 shows a screenshot of the simulation. The horizontal coordinates of the preplanned desired footprints (the method can be used for online planned foot locations as well) were projected onto a three-dimensional ground surface of known geometry. The methods for planning and real-time control were exactly the ones described in sections III and IV. Due to the model assumptions (point mass and point foot), during each stance phase, only the direction CoM-to-foot was feasible for the leg forces, so that the desired eCMP had to be projected via (26) to produce the best feasible leg force. The other two directions were unactuated, so that the robot followed its natural dynamics. To compensate for the lack of controllability, the robot was able to adjust its target location for the subsequent step. Due to the nonlinearity of (26) a numerical forward integration (newton method) of the current state to the time of support transition was performed. Given the estimated state (DCM and CoM position) at the transition, we used the heuristic shown in fig. 7 to continuously compute an appropriate location $r_{f, 2, h}$ for the subsequent foot on the three-dimensional ground surface. The heuristically estimated foot location was commanded to the robot as target location for the foot and used as current foot location $r_{f, 1}$ (see Sec. III) at the support transitions (see step updater block in figure 8).

The stepping time was set to 0.5 seconds. The modeled mass was $60 \mathrm{~kg}$. The average height of the CoM over the ground was set to 0.8 meters (more precisely: $\Delta z_{v r p}=0.8 \mathrm{~m}$ ). The surface height varied between about plus $50 \mathrm{~cm}$ and minus $10 \mathrm{~cm}$. An unknown lateral force of $58.86 \mathrm{~N}$ (correlating to $10 \%$ of the robot's mass) - activated after $2.25 \mathrm{~s}$ and deactivated after $6.75 \mathrm{~s}-$ was perturbing the robot. Additionally, at $t=2.75 \mathrm{~s}$ and $t=3.75 \mathrm{~s}$ the robot suffered lateral and vertical pushes, each resulting in a sudden velocity change of $1 \mathrm{~m} / \mathrm{s}$. The robot was well able to compensate for the perturbations. Before and after the perturbations, the robot was able to track the desired (preplanned) foot locations very well after a couple of steps. Note that in this simulation neither a finite-sized foot nor torques around 


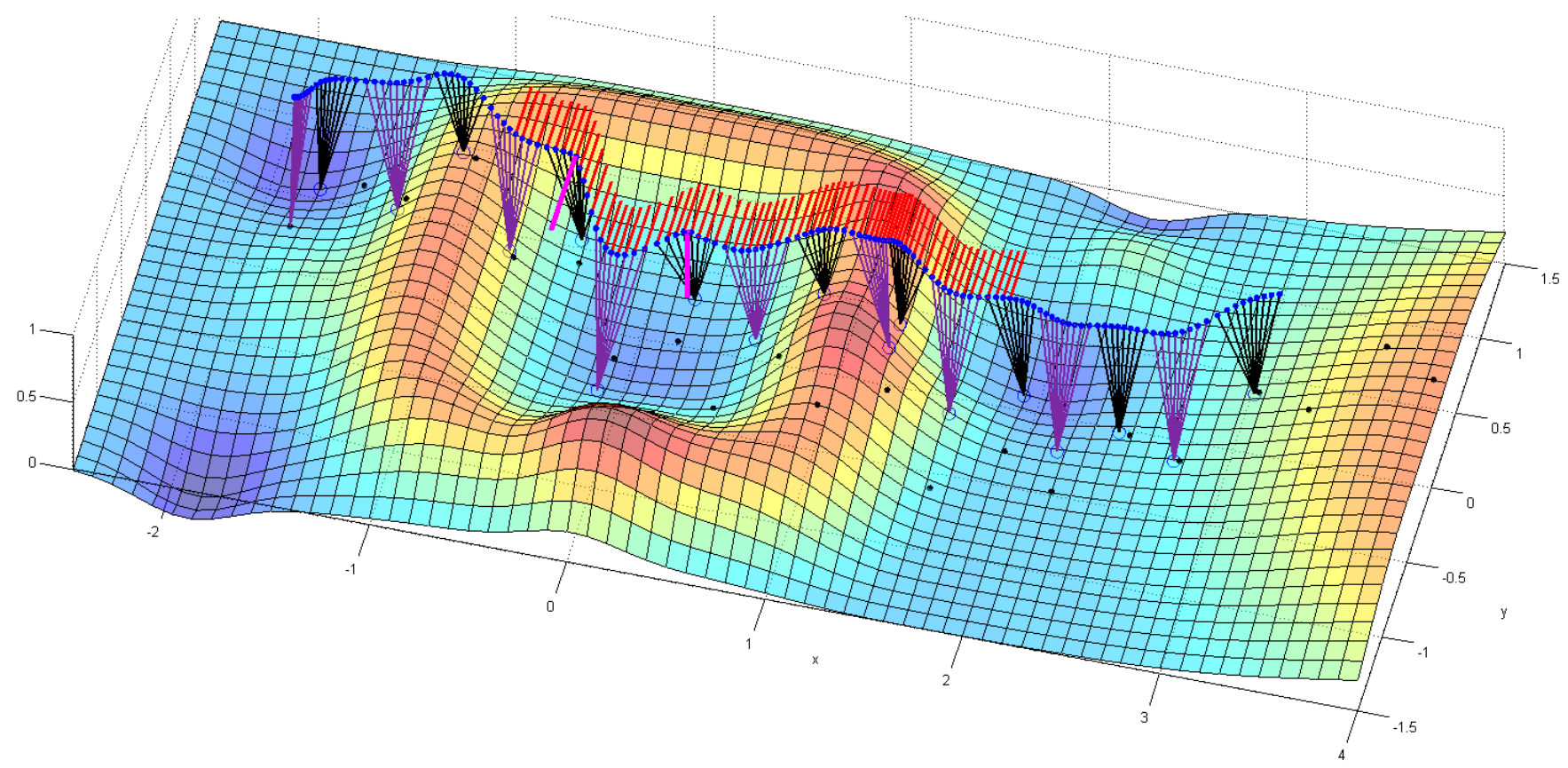

Fig. 9. Prismatic Inverted Pendulum simulation (walking direction: left to right): the biped is modeled as point mass with two point feet (Prismatic Inverted Pendulum model). The red lines denote a lateral force corresponding to $10 \%$ of the robot's mass. The pink lines denote a horizontal and a vertical push, each corresponding to a sudden velocity change of $1 \mathrm{~m} / \mathrm{s}$. The black points on the ground denote the desired (preplanned) footstep locations.

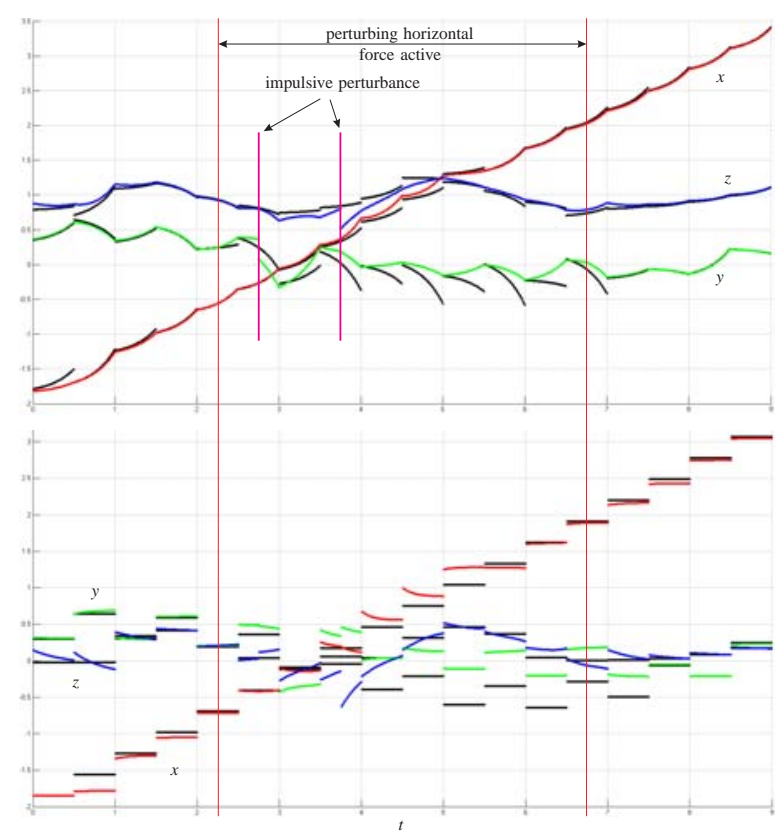

Fig. 10. Prismatic Inverted Pendulum simulation. Top: desired (black) and real DCMs, Bottom: desired (point foot position, black) and real eCMPs

the CoM were used as stabilizing mechanisms, showing the robustness of the proposed underlying control laws. Other simulation setups including (unknown) constant and impulsive perturbing forces in different directions as well as mass estimation errors also showed a very robust performance of the proposed control framework.

\section{B. OpenHRP simulations}

To demonstrate the practicability of the proposed control framework, we performed simulations in OpenHRP (see figures 11 and 12). The robot (a 25-DOF robot called TORO $(75 \mathrm{~kg}$, size: $160 \mathrm{~cm})$ ) walked over a set of stairs, while DCM trajectory planning and feedback control were based on the methods presented in this paper. These simulations can be seen as preliminary work, as the robot was position controlled (admittance-based force control, see [15]) and the step positions were not yet adjusted. Force controlled walking (directly using the joint torques) and step adjustment on the real robot is part of our future work.

\section{CONCLUSION AND FUTURE WORK}

We demonstrated a control framework for bipedal walking on uneven terrain. The derivation of the control laws was facilitated by the linearity of the CoM dynamics. We extended the concept of 'Divergent Component of Motion' (DCM) [13] to 3D and used it to derive methods for planning and tracking three-dimensional DCM trajectories in real-time. We paid specific attention to the feasibility of the finally commanded forces and the problem of underactuation. The capabilities of the control framework were demonstrated in simulations.

Our future work will include the use of the proposed control strategies on our humanoid robot TORO (former DLR Biped [21]). In particular, we are planning to derive methods for force-controlled walking, walking on slopes and uneven ground and push recovery. 


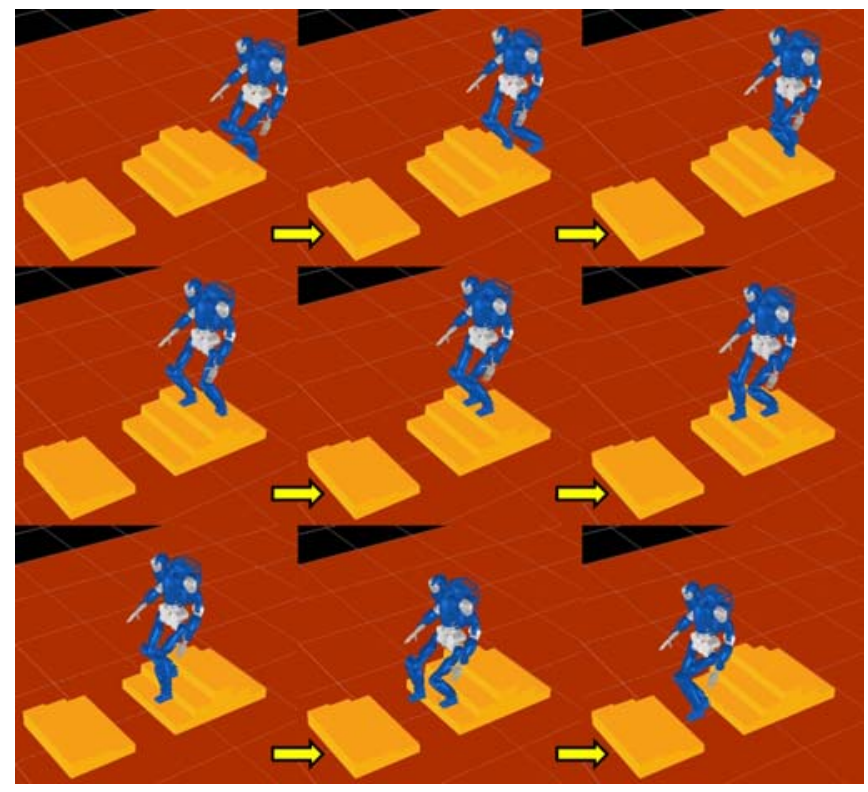

Fig. 11. OpenHRP3 simulation of DLR's bipedal humanoid TORO. Walking over a set of stairs of variable height. (step height differences: $[+12,+12,+12,-12,-12,-12,+10,+5,+3,-18] \mathrm{cm})$

\section{REFERENCES}

[1] P.-B. Wieber, Fast Motions in Biomechanics and Robotics, ser. Lecture Notes in Control and Information Sciences. Springer Berlin / Heidelberg, 2006, vol. 340, ch. Holonomy and Nonholonomy in the Dynamics of Articulated Motion, pp. 411-425.

[2] T. Sugihara, Y. Nakamura, and H. Inoue, "Realtime humanoid motion generation through zmp manipulation based on inverted pendulum control," in IEEE Int. Conf. on Robotics and Automation, 2002, pp. 1404-1409.

[3] M. Vukobratovic and Y. Stepanenko, "On the stability of anthropomorphic systems," Mathematical Biosciences, vol. 15, pp. 1-37, 1972.

[4] B. Stephens and C. Atkeson, "Push recovery by stepping for humanoid robots with force controlled joints," in Humanoid Robots (Humanoids), 2010 10th IEEE-RAS International Conference on, 2010, pp. 52-59.

[5] P.-B. Wieber, "Trajectory free linear model predictive control for stable walking in the presence of strong perturbations," in IEEE-RAS International Conference on Humanoid Robots, 2006.

[6] K. Nishiwaki and S. Kagami, "Online walking control system for humanoids with short cycle pattern generation," The International Journal of Robotics Research, vol. 28, no. 6, pp. 729-742, 2009.

[7] T. Sugihara, "Standing stabilizability and stepping maneuver in planar bipedalism based on the best com-zmp regulator," in IEEE Int. Conf. on Robotics and Automation, 2009, pp. 1966-1971.

[8] S. Kajita, M. Morisawa, K. Miura, S. Nakaoka, K. Harada, K. Kaneko, F. Kanehiro, and K. Yokoi, "Biped walking stabilization based on linear inverted pendulum tracking," in IEEE/RSJ Int. Conference on Intelligent Robots and Systems, 2010, pp. 4489-4496.

[9] A. L. Hof, "The 'extrapolated center of mass' concept suggests a simple control of balance in walking," Human Movement Science, vol. 27, pp. 112-125, 2008.

[10] J. Pratt, J. Carff, S. Drakunov, and A. Goswami, "Capture point: A step toward humanoid push recovery," in IEEE-RAS International Conference on Humanoid Robots, 2006, pp. 200-207.

[11] T. Koolen, T. D. Boer, J. Rebula, A. Goswami, and J. E. Pratt, "Capturability-based analysis and control of legged locomotion. part 1: Theory and application to three simple gait models," IJRR, 2012.

[12] J. E. Pratt, T. Koolen, T. D. Boer, J. Rebula, S. Cotton, J. Carff, M. Johnson, and P. Neuhaus, "Capturability-based analysis and control of legged locomotion, part 2: Application to $\mathrm{m} 2 \mathrm{v} 2$, a lower-body humanoid," IJRR, 2012.



Fig. 12. OpenHRP3 simulation. Top: desired (black) and real DCMs, Bottom: desired (foot centers, black) and real eCMPs

[13] T. Takenaka, T. Matsumoto, and T. Yoshiike, "Real time motion generation and control for biped robot, 1st report: Walking gait pattern generation," in IEEE/RSJ Int. Conference on Intelligent Robots and Systems, 2009, pp. 1084-1091.

[14] J. Englsberger, C. Ott, M. A. Roa, A. Albu-Schäffer, and G. Hirzinger, "Bipedal walking control based on capture point dynamics," in IEEE/RSJ Int. Conference on Intelligent Robots and Systems, 2011, pp. $4420-4427$.

[15] J. Englsberger and C. Ott, "Integration of vertical com motion and angular momentum in an extended capture point tracking controller for bipedal walking," in IEEE-RAS International Conference on Humanoid Robots, 2012, pp. 183-189.

[16] M. Morisawa, S. Kajita, F. Kanehiro, K. Kaneko, K. Miura, and K. Yokoi, "Balance control based on capture point error compensation for biped walking on uneven terrain," in Humanoids, 2012, pp. 734740.

[17] K. Seo, J. Kim, and K. Roh, "Towards natural bipedal walking: Virtual gravity compensation and capture point control," in IEEE/RSJ Int. Conference on Intelligent Robots and Systems, 2012, pp. 4019-4026.

[18] Y. Zhao and L. Sentis, "A three dimensional foot placement planner for locomotion in very rough terrains," in IEEE-RAS International Conference on Humanoid Robots, 2012, pp. 726-733.

[19] J. Slotine and W. Li, "On the adaptive control of robot manipulators," International Journal of Robotics Research, vol. 6, no. 3, pp. 49-59, 1987.

[20] M. B. Popovic, A. Goswami, and H. Herr, "Ground reference points in legged locomotion: Definitions, biological trajectories and control implications," The International Journal of Robotics Research, vol. 24, no. 12, pp. 1013-1032, 2005.

[21] C. Ott, C. Baumgärtner, J. Mayr, M. Fuchs, R. Burger, D. Lee, O. Eiberger, A. Albu-Schäffer, M. Grebenstein, and G. Hirzinger, "Development of a biped robot with torque controlled joints," in IEEERAS International Conference on Humanoid Robots, 2010, pp. 167173 . 